\title{
The prevalence of fatigue among Chinese nursing students in post-COVID-19 era
}

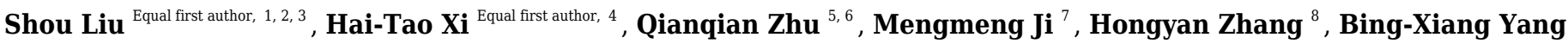 \\ ${ }^{9}$, Wei Bai ${ }^{2,3}$, Hong Cai ${ }^{2,3}$, Yan-Jie Zhao ${ }^{2,3}$, Li Chen $^{4}$, Zong-Mei Ge ${ }^{4}$, Zhiwen Wang $^{7}$, Lin Han $^{8}$, Pan Chen $^{9}$, Shuo \\ Liu $^{9}$, Teris Cheung ${ }^{10}$, Brian J. Hall ${ }^{11,12}$, Feng-rong An ${ }^{\text {Corresp., } 6}$, Yu-Tao Xiang ${ }^{\text {Corresp. 2, } 3}$ \\ 1 Department of Public Health, Medical College, Qinghai University, Xining, Qinghai province, China \\ 2 Centre for Cognitive and Brain Sciences, University of Macau, Macao, China \\ 3 Institute of Advanced Studies in Humanities and Social Sciences, University of Macau, Macao, China \\ 4 Nursing College, Jilin University, Changchun, Jilin province, China \\ 5 School of Nursing, Capital Medical University, Beijing, China \\ 6 The National Clinical Research Center for Mental Disorders \& Beijing Key Laboratory of Mental Disorders Beijing Anding Hospital \& the Advanced \\ Innovation Center for Human Brain Protection, Capital Medical University, School of Mental Health, Beijing, China \\ 7 School of Nursing, Peking University, Beijing, China \\ 8 school of nursing, Lanzhou University, Lanzhou, Gansu province, China \\ 9 School of Health Sciences, Wuhan University, Wuhan, Hubei province, China \\ 10 School of Nursing, Hong Kong Polytechnic University, Hong Kong, China \\ 11 New York University (Shanghai), Shanghai, China \\ 12 Health, Behavior, and Society, Johns Hopkins Bloomberg School of Public Health, Baltimore, USA \\ Corresponding Authors: Feng-rong An, Yu-Tao Xiang \\ Email address: afrylm@sina.com, xyutly@gmail.com
}

Background: Due to the COVID-19 outbreak, all teaching activities in nursing schools were suspended in China, and many nursing students were summoned to work in hospitals to compensate for the shortage of manpower. This study examined the prevalence of fatigue and its association with quality of life (QOL) among nursing students during the post-COVID-19 era in China. Methods: This was a multicenter, cross-sectional study. Nursing students in five Chinese universities were invited to participate. Fatigue, depressive and anxiety symptoms, pain and QOL were measured using standardized instruments. Results: A total of 1,070 nursing students participated. The prevalence of fatigue was $67.3 \%$ (95\% $\mathrm{Cl}: 64.4 \%-70.0 \%)$. Multiple logistic regression analysis revealed that male gender $(\mathrm{P}=0.003, \mathrm{OR}=1.73,95 \% \mathrm{Cl}=1.20-2.49)$, and being a senior nursing student (second year: $\mathrm{OR}=2.20,95 \% \mathrm{Cl}=1.46-3.33, \mathrm{P}<0.001$; third year: $\mathrm{OR}=3.53$, $95 \% \mathrm{Cl}=2.31-5.41, \mathrm{P}<0.001$; and fourth year $\mathrm{OR}=3.59,95 \% \mathrm{Cl}=2.39-5.40, \mathrm{P}<0.001$ ) were significantly associated with more severe fatigue. In addition, participants with more severe depressive $(\mathrm{OR}=1.48,95 \% \mathrm{Cl}=1.22-1.78, \mathrm{P}<0.001)$ and anxiety symptoms $(\mathrm{OR}=1.12,95 \% \mathrm{Cl}=1.05-1.20, \mathrm{P}=0.001)$, and more severe pain $(\mathrm{OR}=1.67$, $95 \% \mathrm{Cl}=1.46-1.91, \mathrm{P}<0.001)$ were significantly associated with more severe fatigue. After 
controlling for covariates, nursing students with fatigue had a lower overall QOL score compared to those without $\left(F_{(1,1070)}=31.4, P<0.001\right)$. Conclusion: Fatigue was common among nursing students in the post-COVID-19 era. Considering the negative impact of fatigue on QOL and daily functioning, routine physical and mental health screening should be conducted for nursing students. Effective stress-reduction measures should be enforced to assist this subpopulation to combat fatigue and restore optimal health. 

the post-COVID-19 era

\section{Running head: fatigue of nursing students}

1,2,3\# Shou Liu, PhD

4\# Hai-Tao Xi, BS,

5,6 Qianqian Zhu, MS

7 Mengmeng Ji, BS

8 Hongyan Zhang MS

9 Bing-Xiang Yang, PhD

2,3 Wei Bai, PhD

2,3 Hong Cai, PhD

2,3 Yan-Jie Zhao, PhD

4 Li Chen, PhD

4 Zong-Mei Ge, MS

7 Zhiwen Wang, PhD

8 Lin Han, PhD

9 Pan Chen, MS

9 Shuo Liu, MS

10 Teris Cheung, PhD

11,12 Brian J. Hall, PhD

6* Fengrong An, MS

2,3* Yu-Tao Xiang, MD, PhD

1. Department of Public Health, Medical College, Qinghai University, Xining, Qinghai province, China;

2. Centre for Cognitive and Brain Sciences, University of Macau, Macao SAR, China;

3. Institute of Advanced Studies in Humanities and Social Sciences, University of Macau, Macao SAR, China;

4. Jilin University Nursing College, Jilin province, China;

5. School of Nursing, Capital Medical University, Beijing, China;

6. The National Clinical Research Center for Mental Disorders \& Beijing Key Laboratory of Mental Disorders Beijing Anding Hospital \& the Advanced Innovation Center for Human Brain Protection, Capital Medical University, 
40 School of Mental Health, Beijing, China;

41 7. Peking University School of Nursing, Beijing, China;

42 8. Lanzhou University school of nursing, Gansu province, China;

43 9. School of Health Sciences, Wuhan University, Hubei province, China;

44 10. School of Nursing, Hong Kong Polytechnic University, Hong Kong SAR, 45 China;

46 11. New York University (Shanghai), Shanghai, China;

47 12. Health, Behavior, and Society, Johns Hopkins Bloomberg School of Public

\# These authors contributed equally to this work.

51

* Address correspondence to Dr. Yu-Tao Xiang, 3/F, Building E12, Faculty of Health Sciences, University of Macau, Avenida da Universidade, Taipa, Macau 54 SAR, China. Fax: +853-2288-2314; Phone: +853-8822-4223; email: 55 xyutly@gmail.com; and Dr. Fengrong An, Beijing Anding Hospital, China; 56 email: afrylm@sina.com 
60

\section{Abstract}

Background: Due to the COVID-19 outbreak, all teaching activities in nursing schools were suspended in China, and many nursing students were summoned to work in hospitals to compensate for the shortage of manpower. This study examined the prevalence of fatigue and its association with quality of life (QOL) among nursing students during the post-COVID-19 era in China.

Methods: This was a multicenter, cross-sectional study. Nursing students in five Chinese universities were invited to participate. Fatigue, depressive and anxiety symptoms, pain and QOL were measured using standardized instruments.

Results: A total of 1,070 nursing students participated. The prevalence of fatigue was $67.3 \%(95 \% \mathrm{CI}: 64.4 \%-70.0 \%)$. Multiple logistic regression analysis revealed that male gender $(P=0.003, O R=1.73,95 \% C I=1.20-2.49)$, and being a senior nursing student (second year: $\mathrm{OR}=2.20,95 \% \mathrm{CI}=1.46$ 3.33, $\mathrm{P}<0.001$; third year: $\mathrm{OR}=3.53,95 \% \mathrm{CI}=2.31-5.41, \mathrm{P}<0.001$; and fourth year $\mathrm{OR}=3.59,95 \% \mathrm{CI}=2.39-5.40, \mathrm{P}<0.001$ ) were significantly associated with more severe fatigue. In addition, participants with more severe depressive $(\mathrm{OR}=1.48,95 \% \mathrm{CI}=1.22-1.78, \mathrm{P}<0.001)$ and anxiety symptoms $(\mathrm{OR}=1.12,95 \% \mathrm{CI}=1.05-1.20, \mathrm{P}=0.001)$, and more severe pain $(\mathrm{OR}=1.67$, $95 \% \mathrm{CI}=1.46-1.91, \mathrm{P}<0.001)$ were significantly associated with more severe fatigue. After controlling for covariates, nursing students with fatigue had a lower overall QOL score compared to those without $\left(F_{(1,1070)}=31.4, P<0.001\right)$. Conclusion: Fatigue was common among nursing students in the postCOVID-19 era. Considering the negative impact of fatigue on QOL and daily functioning, routine physical and mental health screening should be conducted for nursing students. Effective stress-reduction measures should be enforced to assist this subpopulation to combat fatigue and restore optimal health. 
87 Keyword: fatigue, quality of life, nursing students, COVID-19 pandemic 88

89 
90

91

\section{Introduction}

Fatigue refers to abnormal exhaustion following normal activities (Cavanaugh Jr, 2002; Shapiro et al., 2005). Fatigue is associated with lifestyle factors (e.g., physical exertion, lack of sleep, medication use (e.g., antidepressants), physical health problems (e.g., anemia, autoimmune disorders, and chronic obstructive pulmonary disease), and mental health problems (e.g., sleep disorders, anxiety, and depression) (De Venter et al., 2017; Friedberg et al., 2016). All of these factors could lead to additional detrimental outcomes such as headache, faintness, shortness of breath, and increased risk of suicidality (Zhu et al., 2019).

The prevalence of fatigue varies in different populations. For instance, the prevalence of fatigue ranged from $15 \%$ to $30 \%$ in teenagers (Findlay, 2008; Ghandour et al., 2004); $11.9 \%$ in adults, with $8.5 \%$ in men, and $14.9 \%$ in women (Wendt et al., 2019). The prevalence of fatigue was usually more common in certain subpopulations. For instance, the Australian Medical Association found that out of 716 doctors, $53 \%$ were at higher risk of fatigue whilst on duty (Australian Medical Association, 2017). In another study, around $85 \%$ of patients with head and neck cancer experienced fatigue (Bossi et al., 2019). In addition, college students, particularly those enrolled in health-related subjects, often suffered from fatigue (Dol, 2016; Pallant et al., 2020; Shim et al., 2019). For example, one study found that the prevalence of fatigue was $16.5 \%$ among medical students (Tanaka et al., 2008), while the corresponding figure was even higher among nursing students (39.1\%) (de Moraes Amaducci et al., 2010).

Coronavirus Disease 2019 (COVID-19) was first reported in Wuhan, China in December, 2019 (Huang et al., 2020) and then found in more than 200 countries and territories (World Health Organization, 2020). Since April 2020, 
117 COVID-19 has been well-contained in China (National Health Commission of 118 China, 2020). To lower the risk of contagion between students, the spring 119 semester was postponed in all universities in China in early 2020. Further, all 120 classroom teaching was suspended, and replaced by online teaching and

121

122

123

124

125

126

127

128

129

130

131

132

133

134

135

136

137

138

139

140

141

142

143

learning (Xinhuanet, 2020a, 2020b). Due to lockdown measures, outdoor/ physical activities were prohibited in many areas of China. In addition to sudden changes of traditional face-to-face learning modes, students were exposed to high level of academic stress (Brooks et al., 2020; Wang et al., 2020a), which may trigger negative health outcomes including fatigue (Elhai et al., 2020; Király et al., 2020; Miao et al., 2020a; Miao et al., 2020b). Compared to those enrolling in other non-health related subjects, students in health-related majors, such as nursing, may be at higher risks of fatigue due to higher curriculum demand and academic workload from the faculty of nursing.

In order to reduce the likelihood of negative health outcomes caused by fatigue, it is important to understand its prevalence and associated factors. To date, however, fatigue among nursing students in the post- COVID-19 era has not been investigated. Therefore, the aims of this study were to: 1 ) examine the prevalence of fatigue among nursing students in the post COVID-19 era in China and 2) explore the association between fatigue and quality of life (QOL) among nursing students.

\section{Methods}

Participants and study settings

This was a multicenter, cross-sectional study conducted between September 14 and October 7, 2020 across five university nursing schools (Peking University, Capital Medical University, Jilin University, Lanzhou University, and 
144

145

146

147

148

149

150

151

152

153

154

155

156

157

158

159

160

161

162

163

164

165

166

167

168

169

170

171

Wuhan University) in China. In order to avoid contagion during the COVID-19 pandemic, face-to-face interviews were not plausible. Consistent with other studies (Bo et al., 2020; Ma et al., 2020), data were collected using the Questionnaire Star Application embedded in WeChat, which is a widely used social communication application with over 1 billion users in China. WeChat had been used as a teaching tool in the participating nursing schools, therefore, all students were WeChat users. Nursing students in the participating nursing schools were consecutively invited to participate in this study, and those who electronically signed the online written informed consent could access the assessment. To be eligible, participants were 1) undergraduate nursing students, 2) aged between 15 and 28 years, 3) able to understand the content of the assessments, and 4) able to provide written informed consent. The study protocol (No:2020-10) was approved by the Institutional Review Board (IRB) at Beijing Anding Hospital of Capital Medical University and all collaborating university nursing schools.

\section{Measurement tools}

Basic socio-demographic data, such as gender, age, year of study, perceived economic status were collected based on self-report. COVID-19 related experiences were asked using standardized questions, including 1) whether they were volunteers in clinical settings during the COVID-19 pandemic; 2) whether they had negative experiences (e.g., such as verbal abuse and threats) during the COVID-19 pandemic; 3) whether they experienced economic loss during the COVID-19 pandemic; 4) whether they used social media frequently to obtain relevant information during the COVID-19 pandemic; and 5) economic status and perceived health status were also asked using standardized questions.

Fatigue was measured using the numeric rating scale (NRS), scoring from 
172 " 0 " (not suffering from fatigue) to "10" (unbearable suffering from fatigue) 173 (Berger et al., 2010). Higher scores indicated more severe fatigue, and a score 174 of $\geqslant 4$ was considered "clinically relevant fatigue" ("having fatigue" hereafter) 175 (Oldenmenger et al., 2013). Another NRS was adopted to evaluate severity of 176 overall body pain (pain hereafter)(Haefeli and Elfering, 2006), which was 177 scored from "0" (no pain) to "10" (worst pain imaginable), with a higher score 178 indicating more severe pain ( Li et al., 2009; Li et al., 2007; Liu and Li, 2004). 179 The Chinese version of the Patient Health Questionnaire (PHQ-2) was used 180 to measure depressive symptoms (Chen et al., 2015; Kroenke et al., 2001) in 181 the past week. Each item scored from 0 (not at all) to 3 (nearly every day). 182 The total score ranged from 0 to 6 , with a higher score representing more severe depressive symptoms. The Chinese version of the Generalized Anxiety Disorder scale seven items (GAD-7) was used to assess anxiety symptoms. Each item scored from 0 (absolutely no) to 3 (almost every day), with a higher score indicating more severe anxiety symptoms (He et al., 2010; Spitzer et al., 2006). The overall QOL was measured using the first two items on overall QOL of the World Health Organization Quality of Life-brief version (WHOQOLBREF) (Fang, 1999; Harper et al., 1998; Xia et al., 2012), with higher scores indicating greater QOL.

\section{Statistical analysis}

Data were analyzed using the IBM Statistical Package for Social Science (SPSS) program, version 24.0. The comparisons between nursing students with and without fatigue were conducted using two independent samples $t$ tests, Mann-Whitney U Tests, and Chi-square tests, as appropriate. Analysis of covariance (ANCOVA) was conducted to examine the independent association between fatigue and $\mathrm{QOL}$, after adjusting for variables with significant group differences in univariate analyses. Binary logistic regression analysis with the "enter" method was performed to test the independent 
201 correlates of fatigue, with fatigue as the dependent variable, and those with 202 significant group differences in the univariate analyses as independent 203 variables. Significance level was set at 0.05 (two-tailed).

204

205

206

\section{Results}

Altogether, 1,121 nursing students were consecutively invited to participate 207 in this study; of whom, 1,070 met the study criteria and completed the assessment, yielding a response rate of $95.5 \%$. The prevalence of fatigue was $67.3 \%$ (95\%CI: 64.4\%-70.0\%). There were significant differences between fatigue and no fatigue groups in terms of gender, age, year of study, economic loss during COVID-19 pandemic, financial perception, health perception, and the PHQ-2, GAD-7, and pain total scores (Table 1). After controlling for covariates, nursing students with fatigue had lower $\mathrm{QOL}\left(\mathrm{F}_{(1,1070)}=31.4\right.$, $P<0.001)$ than those without.

Multiple logistic regression analysis revealed that men (Odds Ratio $(O R)=1.73,95 \% C I=1.20-2.49, P=0.003)$, students in their $2^{\text {nd }}(O R=2.20$, $95 \% \mathrm{CI}=1.46-3.33, P<0.001), 3^{\text {rd }}(\mathrm{OR}=3.53,95 \% \mathrm{CI}=2.31-5.41, P<0.001)$ and $4^{\text {th }}$ year $(\mathrm{OR}=3.59,95 \% \mathrm{CI}=2.39-5.40, P<0.001$; compared to students 219 in their first year), moderate economic loss during the COVID-19 pandemic ( $\mathrm{OR}=1.48,95 \% \mathrm{CI}=1.08-3.33, P=0.015$; compared to low loss), more severe depressive $(\mathrm{OR}=1.48,95 \% \mathrm{CI}=1.22-1.78, P<0.001)$, and anxiety symptoms $(\mathrm{OR}=1.12,95 \% \mathrm{CI}=1.05-1.20, P=0.001)$, and more severe pain $(\mathrm{OR}=1.67$, $95 \% \mathrm{CI}=1.46-1.91, P<0.001)$ were significantly associated with more severe fatigue (Table 2).

\section{Discussion}

This study examined the prevalence of fatigue among nursing students in post-COVID-19 era. We found that $67.3 \%$ of nursing students reported 
229 fatigue, which is almost double the prevalence of fatigue (36\%) in qualified 230 nurses on shift work assessed by the Occupational Fatigue Exhaustion 231 Recovery scale (Geiger-Brown et al., 2012). Our finding was similar to the 232 corresponding figure $(73.7 \%)$ in frontline staff (including doctors, nurses, 233 police officers, volunteers, community workers, and journalists) during 234 COVID-19 outbreak in China as measured by the Fatigue Self-Assessment Scale (Teng et al., 2020). In contrast, the level of fatigue among medical students was relatively low (13.8\%) before the COVID-19 outbreak (Abdali et al., 2020). Owing to different measurement tools on fatigue, direct comparisons between studies should be interpreted with caution.

Fatigue appeared to be common among nursing students in the postCOVID-19 era and this can be attributed to several reasons. First, previous studies found that fatigue among students who majored in health-related subjects was usually related to poor academic performance and related problems, such as absenteeism, and having a sedentary lifestyle (e.g., lack of physical exercise) (Cruz et al., 2018). Sudden shifting from traditional classroom learning to online learning coupled with limited outdoor physical activities during the COVID-19 outbreak in China may have led to poorer academic performance, and increased absenteeism, which is often linked with sedentary lifestyle, and this in turn may have led to more fatigue among nursing students. Second, many nursing and medical students served as volunteers in clinical settings during the COVID-19 outbreak. Persistent high levels of stress and anxiety (Cao et al., 2020) at work could further exacerbate the risk of fatigue (Abdali et al., 2020; Doerr et al., 2015; Nijrolder et al., 2008). In addition, potential risk of susceptibility to COVID-19 infection on top of a heavy clinical workload may have also escalated the risk of fatigue amidst the COVID-19 outbreak. Third, daily infection precautionary measures at work (e.g., face mask wearing, frequent hand-washing, full gear personal 
257 protection equipment adherence), reduced social etiquette practices (e.g., 258 shaking hands) and social distancing, could lead to boredom (Miao et al., 259 2020b), anxiety, frustration (Aristovnik et al., 2020), and mental fatigue.

260 In this study, we found that male students were more likely to report 261 fatigue than their female counterparts. In China, nursing students are 262 predominantly women. In traditional Chinese culture, men have been ascribed 263 the social status of "pillars" within the family and in the society; therefore, 264 they were often expected to be responsible for more heavy tasks and 265 challenges than women in public health crisis situations (e.g., COVID-19 266 outbreak). In addition, female students who major in health-related subjects usually have a better academic performance than male students (Alzahrani et al., 2018; Voyer and Voyer, 2014). Such gender differences in academic performance suggests that female students may adapt better than male students in the switching of learning modes. These social and educational factors could result in greater fatigue in male students. Similar to previous findings (Labrague and Ballad, 2020), we found that the $2^{\text {nd }}(O R=2.20), 3^{\text {rd }}$ $(O R=3.53)$ and $4^{\text {th }}$ year students $(O R=3.59)$ were more likely to report fatigue than $1^{\text {st }}$ year students. Senior nursing students receive more crisis response and medical training compared to junior students. As such, they usually undertook a greater responsibility in combating the COVID-19 outbreak, which possibly explained the differences in the level of fatigue between years of study.

279 There were independent associations between fatigue, depression, anxiety and pain. More severe fatigue was associated with more severe depression, anxiety and more severe pain among nursing students in this study. Similar findings were found in university students (Shim et al., 2019) before and during COVID-19 outbreak (Verma, 2020; Wang et al., 2020b). 
284 The relationship between fatigue and depression / anxiety were bidirectional 285 (Thorsteinsson et al., 2019) (i.e., fatigue could increase the risk of depression 286 and anxiety, and vice versa). Consistent with previous findings (Kaasa et al., 1999; Yoon et al., 2019), in this study more severe pain was associated with

288

289

290

291

292

293

294

295

296

297

298

299

300

301

302

303

304

305

306

307

308

309 310

a higher risk of fatigue in nursing students. Pain is defined as an unpleasant sensory and emotional experience usually associated with actual or potential tissue damage (Raja et al., 2020) caused by internal and/or external factors (e.g. cold, heat, physical pressure and lesions). Adjustment mechanisms in the human body attempt to relieve pain through the central brain feedback system (Mauger, 2013). If the predisposing factors cannot be addressed and remain chronic, the adjustment/restoration system will be out of balance and the body will be fatigued (Aaronson et al., 1999; Sharpe and Wilks, 2002).

Similar to previous findings (Kratz et al., 2017; Nunes et al., 2017), we found that nursing students with fatigue had a lower overall QOL than those without. As a widely used health outcome measure, QOL is closely associated with the interactions between protective factors (e.g., better social support) and risk factors (e.g., physical distress) (Hatoum et al., 1998). Fatigue was also associated with physical and mental distress, which could lower QOL.

The strengths of this study included the multi-site design, relatively large sample size and use of standardized instruments. However, several methodological limitations should be acknowledged. First, casual relationships between fatigue and other variables could not be established due to crosssectional design. Second, only five university nursing schools were included, and hence, our findings may not be generalizable to all nursing students in China. Third, some factors (e.g., academic pressure and social support) associated with fatigue were not assessed due to logistical reasons. 
311

312

313

314

315

316

317

318

319

320

321

322

323

324

325

326

327

328

329

330

331

332

333

334

335

336

337

Conclusion

Fatigue was common among nursing students in post-COVID-19 era. Considering the negative impact of fatigue on QOL and daily functioning, routine physical and mental health screening should be conducted for nursing students. Effective stress-reduction strategies should be executed to assist nursing students to combat fatigue and restore optimal health.

\section{Acknowledgements}

None.

\section{Contributors}

Study design: Shou Liu, Feng-Rong An, Yu-Tao Xiang.

Data collection, analysis and interpretation: Qianqian Zhu, Zhiwen Wang, Lin Han, Pan Chen, Wei Bai, Hong Cai, Yan-Jie Zhao, Li Chen, Zong-Mei Ge, Mengmeng Ji, Hongyan Zhang, Bing-Xiang Yang, Shuo Liu.

Drafting of the manuscript: Shou Liu, Teris Cheung, Yu-Tao Xiang.

Critical revision of the manuscript: Brian J. Hall.

Approval of the final version for publication: all co-authors.

\section{Funding statement}

The study was supported by the National Science and Technology Major Project for investigational new drug (2018ZX09201-014), the Beijing Municipal Science \& Technology Commission (No. Z181100001518005), the 2020 Higher Education Teaching Achievement Cultivation Project of Gansu Province, the Fundamental Research Funds for the Central Universities (2020YJ065), the University of Macau (MYRG2019-00066-FHS) and the National Natural Science Foundation of China(81860606). 
339 Ethics approval statement

340 The study protocol was approved by the Institutional Review Board (IRB) at 341 Beijing Anding Hospital of Capital Medical University and all collaborating 342 university nursing schools.

343

344 Conflict of interest statement

345 All authors declare that there are no conflicts of interest. 


\section{References}

Aaronson, L.S., Teel, C.S., Cassmeyer, V., Neuberger, G.B., Pallikkathayil, L., Pierce, J., Press, A.N., Williams, P.D., Wingate, A., 1999. Defining and measuring fatigue. Image: the journal of nursing scholarship 31 (1), 45-50.

Abdali, N., Nobahar, M., Ghorbani, R., 2020. Evaluation of emotional intelligence, sleep quality, and fatigue among Iranian medical, nursing, and paramedical students: A cross-sectional study. Qatar Med J 2019 (3), 15.

Alzahrani, S.S., Soo Park, Y., Tekian, A., 2018. Study habits and academic achievement among medical students: A comparison between male and female subjects. Medical Teacher 40 (sup1), S1-S9.

Aristovnik, A., Keržič, D., Ravšelj, D., Tomaževič, N., Umek, L., 2020. Impacts of the COVID-19 pandemic on life of higher education students: A global perspective. Sustainability 12 (20), 8438.

Australian Medical Association, 2017. Managing the risks of fatigue in the medical workforce 2016: AMA safe hours audit. Barton,ACT:AMA.

Berger, A.M., Abernethy, A.P., Atkinson, A., Barsevick, A.M., Breitbart, W.S., Cella, D., Cimprich, B., Cleeland, C., Eisenberger, M.A., Escalante, C.P., Jacobsen, P.B., Kaldor, P., Ligibel, J.A., Murphy, B.A., O'Connor, T., Pirl, W.F., Rodler, E., Rugo, H.S., Thomas, J., Wagner, L.I., 2010. NCCN Clinical Practice Guidelines Cancer-related fatigue. Journal Natl Compr Canc Netw 8 (8), 904-931.

Bo, H.X., Li, W., Yang, Y., Wang, Y., Zhang, Q., Cheung, T., Wu, X., Xiang, Y.T., 2020. Posttraumatic stress symptoms and attitude toward crisis mental health services among clinically stable patients with COVID-19 in China. Psychol Med, 1-2.

Bossi, P., Di Pede, P., Guglielmo, M., Granata, R., Alfieri, S., lacovelli, N.A., Orlandi, E., Guzzo, M., Bianchi, R., Ferella, L., Laryngology, 2019. Prevalence of Fatigue in Head and Neck Cancer Survivors. Annals of Otology, Rhinology 128 (5), 413-419.

Brooks, S.K., Webster, R.K., Smith, L.E., Woodland, L., Wessely, S., Greenberg, N., Rubin, G.J., 2020. The psychological impact of quarantine and how to reduce it: rapid review of the evidence. The Lancet 395 (10227), 912-920.

Cao, W., Fang, Z., Hou, G., Han, M., Xu, X., Dong, J., Zheng, J., 2020. The psychological impact of the COVID-19 epidemic on college students in China. Psychiatry research, 112934.

Cavanaugh Jr, R.M., 2002. Evaluating adolescents with fatigue: ever get tired of it? Pediatrics in review 23 (10), 9.

Chen, M., Sheng, L., Qu, S., 2015. Diagnostic test of screening depressive disorder in general hospital with the Patient Health Questionnaire (in Chinese). Chinese Mental Health 29 (4), 241-245.

Cruz, J.P., Felicilda-Reynaldo, R.F.D., Lam, S.C., Contreras, F.A.M., Cecily, H.S.J., Papathanasiou, I.V., Fouly, H.A., Kamau, S.M., Valdez, G.F.D., Adams, K.A., 2018. Quality of life of nursing students from nine countries: A cross-sectional study. Nurse Education Today 66, 135-142.

de Moraes Amaducci, C., de Correa Mota, D.D.F., de Mattos Pimenta, C.A., 2010. Fatigue among nursing undergraduate students. J Revista da Escola de Enfermagem da USP 44 (4), 1052-1058.

De Venter, M., Illegems, J., Van Royen, R., Moorkens, G., Sabbe, B.G., Van Den Eede, F., 2017. Differential effects of childhood trauma subtypes on fatigue and physical functioning in chronic fatigue syndrome. Comprehensive Psychiatry 78, 76-82.

Doerr, J.M., Ditzen, B., Strahler, J., Linnemann, A., Ziemek, J., Skoluda, N., Hoppmann, C.A., Nater, U.M., 2015. Reciprocal relationship between acute stress and acute fatigue in everyday life in a sample of university students. Biological psychology 110, 42-49.

Dol, K.S., 2016. Fatigue and pain related to Internet usage among university students. Journal of physical therapy science 28 (4), 1233-1237.

Peer) reviewing PDF | (2021:01:56906:1:1:NEW 23 Feb 2021) 
Elhai, J.D., Yang, H., McKay, D., Asmundson, G.J.G., 2020. COVID-19 anxiety symptoms associated with problematic smartphone use severity in Chinese adults. J Affect Disord 274, 576-582.

Fang, J.Q., \& Hao, Y. A. , 1999. Reliability and validity for Chinese version of WHO Quality of Life Scale (in Chinese). Chinese Mental Health Journal 13 (4), 203-209.

Findlay, S.M., 2008. The tired teen: A review of the assessment and management of the adolescent with sleepiness and fatigue. Paediatr Child Health 13 (1), 37-42.

Friedberg, F., Adamowicz, J.L., Caikauskaite, I., Napoli, A., Shapira, O., Hobbs, M., Bromet, E., Kotov, R., Gonzalez, A., Clouston, S., 2016. Fatigue severity in World Trade Center (9/11) responders: a preliminary study. Fatigue: Biomedicine, Health \& Behavior 4 (2), 70-79.

Geiger-Brown, J., Rogers, V.E., Trinkoff, A.M., Kane, R.L., Bausell, R.B., Scharf, S.M., 2012. Sleep, sleepiness, fatigue, and performance of 12-hour-shift nurses. Chronobiol Int 29 (2), 211-219.

Ghandour, R.M., Overpeck, M.D., Huang, Z.J., Kogan, M.D., Scheidt, P.C., Medicine, A., 2004. Headache, stomachache, backache, and morning fatigue among adolescent girls in the United States: associations with behavioral, sociodemographic, and environmental factors. J Archives of Pediatrics 158 (8), 797-803.

Haefeli, M., Elfering, A., 2006. Pain assessment. European Spine Journal 15 (1), S17-S24.

Harper, A., Power, M., Grp, W., 1998. Development of the World Health Organization WHOQOL-BREF quality of life assessment. Psychological Medicine 28 (3), 551-558.

Hatoum, H.T., Kong, S.X., Kania, C.M., Wong, J.M., Mendelson, W.B., 1998. Insomnia, health-related quality of life and healthcare resource consumption. Pharmacoeconomics 14 (6), 629-637.

He, X.Y., Li, C.B., Qian, J., Cui, H.S., Wu, W.Y., 2010. Reliability an dvalidity of a generalized anxiety disorder scale in general haspital outpatients. Shanghai Archives of Psychiatry(4), 200-203.

Huang, C., Wang, Y., Li, X., Ren, L., Zhao, J., Hu, Y., Zhang, L., Fan, G., Xu, J., Gu, X., 2020. Clinical features of patients infected with 2019 novel coronavirus in Wuhan, China. The lancet 395 (10223), 497-506.

Kaasa, S., Loge, J.H., Knobel, H., Jordhøy, M., Brenne, E., 1999. Fatigue. Measures and relation to pain. Acta Anaesthesiologica Scandinavica 43 (9), 939-947.

Király, O., Potenza, M.N., Stein, D.J., King, D.L., Hodgins, D.C., Saunders, J.B., Griffiths, M.D., Gjoneska, B., Billieux, J., Brand, M., 2020. Preventing problematic internet use during the COVID-19 pandemic: Consensus guidance. Comprehensive Psychiatry, 152180.

Kratz, A.L., Braley, T.J., Foxen-Craft, E., Scott, E., Murphy, J.F., 3rd, Murphy, S.L., 2017. How Do Pain, Fatigue, Depressive, and Cognitive Symptoms Relate to Well-Being and Social and Physical Functioning in the Daily Lives of Individuals With Multiple Sclerosis? Arch Phys Med Rehabil 98 (11), 2160-2166.

Kroenke, K., Spitzer, R.L., Williams, J.B., 2001. The PHQ-9: validity of a brief depression severity measure. Journal of general internal medicine 16 (9), 606-613.

Labrague, L., Ballad, C.A., 2020. lockdown fatigue among college students during the covid-19 pandemic: predictive role of personal resilience, coping behaviours, and health. medRxiv.

Li, L., Herr, K., Chen, P., 2009. Postoperative pain assessment with three intensity scales in Chinese elders. Journal of Nursing Scholarship 41 (3), 241-249.

Li, L., Liu, X., Herr, K., 2007. Postoperative pain intensity assessment: a comparison of four scales in Chinese adults. Pain Medicine 8 (3), 223-234.

Liu, X.-Q., Li, L., 2004. The selection of pain intensity assessment scales in Chinese elders (in Chinese). Chinese Journal of Nursing 39(3), 165-167. 
Ma, Y.F., Li, W., Deng, H.B., Wang, L., Wang, Y., Wang, P.H., Bo, H.X., Cao, J., Wang, Y., Zhu, L.Y., Yang, Y., Cheung, T., $\mathrm{Ng}, \mathrm{C} . \mathrm{H} ., \mathrm{Wu}, \mathrm{X} ., \mathrm{Xiang}, \mathrm{Y} . T ., 2020$. Prevalence of depression and its association with quality of life in clinically stable patients with COVID-19. J Affect Disord 275, 145-148.

Mauger, A.R., 2013. Fatigue is a pain-the use of novel neurophysiological techniques to understand the fatiguepain relationship. Frontiers in physiology 4, 104.

Miao, C., Xueming, C., Tour, L., Haibo, Y., Hall, B.J., 2020a. Media use and acute psychological outcomes during COVID-19 outbreak in China. Journal of Anxiety Disorders 74, 102248.

Miao, C., Xueming, C., Tour, L., Haibo, Y., Hall, B.J., 2020b. Psychological distress and state boredom during the COVID-19 outbreak in China: the role of meaning in life and media use. European Journal of Psychotraumatology 11 (1), 1769379.

National Health Commission of China, 2020. Interpretation of the circular on further strengthening the work of infection prevention and control in medical institutions in order to implement the requirements of regular epidemic prevention and control (in Chinese), National Health Commission of China, Available on:http://www.nhc.gov.cn/yzygi/s3594/202005/4f8326122836421c9d50bf1a074402ee.shtml.

Nijrolder, I., van der Horst, H., van der Windt, D., 2008. Prognosis of fatigue. A systematic review. J Psychosom Res 64 (4), 335-349.

Nunes, M.D.R., Jacob, E., Bomfim, E.O., Lopes-Junior, L.C., de Lima, R.A.G., Floria-Santos, M., Nascimento, L.C., 2017. Fatigue and health related quality of life in children and adolescents with cancer. Eur J Oncol Nurs 29, 3946.

Oldenmenger, W.H., de Raaf, P.J., de Klerk, C., van der Rijt, C.C., 2013. Cut points on 0-10 numeric rating scales for symptoms included in the Edmonton Symptom Assessment Scale in cancer patients: a systematic review. Journal Pain Symptom Manage 45 (6), 1083-1093.

Pallant, A., Sullivan, T., Kaluzny, A., 2020. Nutritional deficiency presenting as acute pain, fatigue and bruising in a college health clinic. J Am Coll Health, 1-3.

Rainville, P., 2002. Brain mechanisms of pain affect and pain modulation. Current opinion in neurobiology 12 (2), 195-204.

Raja, S.N., Carr, D.B., Cohen, M., Finnerup, N.B., Flor, H., Gibson, S., Keefe, F.J., Mogil, J.S., Ringkamp, M., Sluka, K.A., 2020. The revised International Association for the Study of Pain definition of pain: concepts, challenges, and compromises. Pain 161 (9), 1976-1982.

Shapiro, C., Ohayon, M., Huterer, N., Grunstein, R., 2005. Fighting fatigue and sleepiness. Practical strategies for minimizing sleepiness and fatigue. Joli joco publications Inc., Ontario.

Sharpe, M., Wilks, D., 2002. Fatigue. Bmj 325 (7362), 480-483.

Shim, E.-J., Noh, H.-I., Yoon, J., Mun, H.S., Hahm, B.-J.J.J.o.A.c.h., 2019. A longitudinal analysis of the relationships among daytime dysfunction, fatigue, and depression in college students. Journal of American college health 67 (1), 51-58.

Spitzer, R.L., Kroenke, K., Williams, J.B., Löwe, B., 2006. A brief measure for assessing generalized anxiety disorder: the GAD-7. Arch Intern Med 166 (10), 1092-1097.

Tanaka, M., Mizuno, K., Fukuda, S., Shigihara, Y., Watanabe, Y., 2008. Relationships between dietary habits and the prevalence of fatigue in medical students. Nutrition 24 (10), 985-989.

Teng, Z., Wei, Z., Qiu, Y., Tan, Y., Chen, J., Tang, H., Wu, H., Wu, R., Huang, J., 2020. Psychological status and fatigue of frontline staff two months after the COVID-19 pandemic outbreak in China: A cross-sectional study. J 
Affect Disord 275, 247-252.

Thorsteinsson, E.B., Brown, R.F., Owens, M.T., disease, m., 2019. Modeling the effects of stress, anxiety, and depression on rumination, sleep, and fatigue in a nonclinical sample. The Journal of nervous 207 (5), 355359.

Verma, K., 2020. The mental health impact of the COVID-19 epidemic on college students in India. Asian Journal of Psychiatry.

Voyer, D., Voyer, S.D., 2014. Gender differences in scholastic achievement: a meta-analysis. Psychological bulletin 140 (4), 1174-1204.

Wang, G., Zhang, Y., Zhao, J., Zhang, J., Jiang, F., 2020a. Mitigate the effects of home confinement on children during the COVID-19 outbreak. Lancet 395 (10228), 945-947.

Wang, Z.-H., Yang, H.-L., Yang, Y.-Q., Liu, D., Li, Z.-H., Zhang, X.-R., Zhang, Y.-J., Shen, D., Chen, P.-L., Song, W.-Q., 2020b. Prevalence of anxiety and depression symptom, and the demands for psychological knowledge and interventions in college students during COVID-19 epidemic: A large cross-sectional study. J Affect Disord 275, 188-193.

Wendt, A., Costa, C.S., Machado, A.K.F., Costa, F.S., Neves, R.G., Flores, T.R., Santos, I., Wehrmeister, F.C., 2019. Sleep disturbances and daytime fatigue: data from the Brazilian National Health Survey, 2013. Cadernos de saude publica 35 , e00086918.

World Health Organization, 2020. Weekly operational update on COVID-19 - 30 October 2020. World Health Organization, Available on: https://www.who.int/publications/m/item/weekly-operational-update---30october-2020.

Xia, P., Li, N., Hau, K.-T., Liu, C., Lu, Y., 2012. Quality of life of Chinese urban community residents: a psychometric study of the mainland Chinese version of the WHOQOL-BREF. BMC Medical Research Methodology 12 (1), 37.

Xinhuanet, 2020a. China Focus: Schools start online courses as epidemic control postpones new semester http://www.xinhuanet.com/english/2020-02/17/c 138792006.htm (access March 1st 2020).

Xinhuanet, 2020b. China postpones school semester amid novel coronavirus outbreak. http://www.xinhuanet.com/english/2020-01/28/c 138738646.htm (access March 1st 2020).

Yoon, I.A., Sturgeon, J.A., Feinstein, A.B., Bhandari, R.P., 2019. The role of fatigue in functional outcomes for youth with chronic pain. European Journal of Pain 23 (8), 1548-1562.

Zhu, H., Han, G., Li, S., 2019. Chronic Fatigue Stress and Sudden Death, Effects of Stress on Human Health. IntechOpen. 


\section{Table 1 (on next page)}

Socio-demographical and scale' scores of nursing students 
1 Table 1. Socio-demographical and scale' scores of nursing students

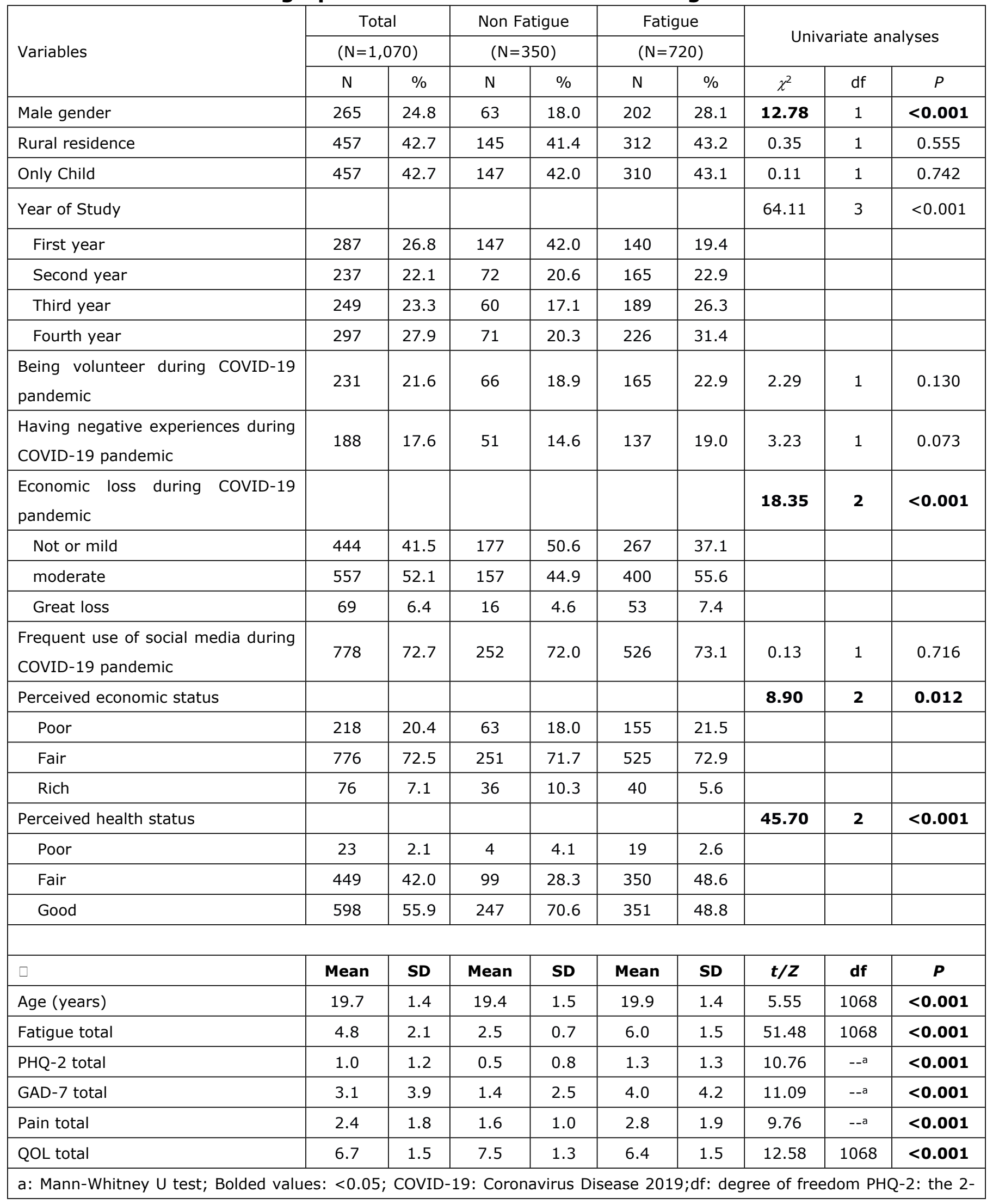


item Patient Health Questionnaire; QOL: quality of life; GAD-7: 7-item Generalized Anxiety Disorder Scale; SD: standard deviation. 
Table 2 (on next page)

Independent correlates of fatigue by multiple logistic regression analysis 
1 Table 2 Independent correlates of fatigue by multiple logistic regression 2 analysis

\begin{tabular}{|c|c|c|c|}
\hline \multirow{2}{*}{ Variables } & \multicolumn{3}{|c|}{ Multiple logistic regression analysis } \\
\hline & $P$ & OR & $95 \% \mathrm{CI}$ \\
\hline Male gender & 0.003 & 1.73 & $1.20-2.49$ \\
\hline \multicolumn{4}{|l|}{ Year of study } \\
\hline Frist year & - & 1.0 & - \\
\hline Second year & $<0.001$ & 2.20 & $1.46-3.33$ \\
\hline Third year & $<0.001$ & 3.53 & 2.31-5.41 \\
\hline Fourth year & $<0.001$ & 3.59 & 2.39-5.40 \\
\hline \multicolumn{4}{|c|}{ Economic loss during COVID-19 pandemic } \\
\hline Not or mild & - & 1.0 & - \\
\hline Moderate & 0.015 & 1.48 & $1.08-2.02$ \\
\hline Great loss & 0.352 & 1.41 & $0.68-2.91$ \\
\hline \multicolumn{4}{|c|}{ Perceived economic status } \\
\hline Poor & - & 1.0 & - \\
\hline Fair & 0.100 & 1.41 & $0.94-2.13$ \\
\hline Rich & 0.495 & 1.26 & $0.65-2.44$ \\
\hline \multicolumn{4}{|c|}{ Perceived health status } \\
\hline Poor & - & 1.0 & - \\
\hline Fair & 0.151 & 2.70 & $0.70-10.50$ \\
\hline Good & 0.312 & 2.02 & $0.52-7.86$ \\
\hline PHQ-2 total & $<0.001$ & 1.48 & $1.22-1.78$ \\
\hline GAD-7 total & 0.001 & 1.12 & $1.05-1.20$ \\
\hline Pain total & $<0.001$ & 1.67 & $1.46-1.91$ \\
\hline \multicolumn{4}{|c|}{$\begin{array}{l}\text { Bolded values: }<0.05 ; \mathrm{CI} \text { : confidential interval; OR: odds ratio; PHQ-2: the 2-item Patient Health } \\
\text { Questionnaire; QOL: quality of life; GAD-7: 7-item Generalized Anxiety Disorder Scale. There was } \\
\text { collinearity between age and grade, therefore age was not entered in the model as an independent } \\
\text { variable. }\end{array}$} \\
\hline
\end{tabular}

